\title{
ON THE APPROXIMATION OF FINSLER METRICS ON EUCLIDEAN DOMAINS
}

\author{
by G. BARBATIS
}

(Received 3rd December 1997)

\begin{abstract}
We prove that Finsler metrics on Euclidean domains can be approximated in a certain sense by so-called Finsler-type metrics. As an application we improve upon previous estimates on the fundamental solution of higher order parabolic equations.
\end{abstract}

1991 Mathematics subject classification: $35 \mathrm{~K} 35,53 \mathrm{C} 60$.

\section{Introduction}

The aim of this paper is to prove that Finsler metrics on Euclidean domains can be approximated, in a way to be made precise, by a certain class of Finsler-type metrics. While this is a geometrical problem, it is motivated by a problem in the spectral theory of higher order elliptic operators. We describe that first.

Let $H$ be a self-adjoint elliptic differential operator of the form

$$
H f(x)=(-1)^{m} \sum_{\substack{|x|=m \\|\beta|=m}} D^{\alpha}\left\{a_{\alpha \beta}(x) D^{\beta} f(x)\right\},
$$

acting on a Euclidean domain $\Omega$ and subject to Dirichlet boundary conditions on $\partial \Omega$. Let $a(x, \xi)=\sum a_{\alpha \beta}(x) \xi^{\alpha+\beta}, x \in \Omega, \xi \in \mathbf{R}^{N}$, be its principal symbol. Under some regularity assumptions (see definitions below) there is canonically associated with the symbol a Finsler metric $d(x, y)$ given by

$$
d(x, y)=\sup \{\phi(y)-\phi(x) \mid \phi \in \mathcal{E}\}
$$

where $\mathcal{E}$ is the class of all Lipschitz continuous functions $\phi$ on $\Omega$ that satisfy $a(z, \nabla \phi(z)) \leq 1$ for a.e. $z \in \Omega$. This metric is of importance in the study of the associated parabolic equation

$$
\frac{\partial u}{\partial t}(x, t)=-H u(x, t), \quad x \in \Omega, t>0
$$

It is proved in [18] that if $\Omega=\mathbf{R}^{N}$, if the coefficients $\left\{a_{x \beta}\right\}$ are real and smooth and if 
the symbol $a(x, \xi)$ satisfies the so-called strong convexity condition (see Definition 3 ) then the fundamental solution $K(t, x, y)$ of (2) has short-time asymptotics given by

$$
K(t, x, y) \sim t^{-N / 2 m} \exp \left\{-\sigma_{m} \frac{d(x, y)^{2 m /(2 m-1)}}{t^{1 /(2 m-1)}}\right\}, \quad(t \rightarrow 0)
$$

provided $x$ and $y$ are close enough. Here

$$
\sigma_{m}=(2 m-1)(2 m)^{-2 m /(2 m-1)} \sin (\pi / 4 m-2)
$$

In fact the formula obtained in [18] also contains some subexponential terms, which we have omitted here.

While heat kernel estimates for higher-order operators on Euclidean domains or manifolds have been studied for many years (see [11] for a detailed account), the problem of obtaining sharp bounds has only been addressed recently. The estimates obtained in [4] are valid for operators with measurable coefficients and were obtained by means of comparison with the model operator $(-\Delta)^{m}$. They are sharp as long as one restricts one's attention to the Euclidean distance. That paper was followed by [5], where it is shown that if $H$ is an operator of the above form acting on an arbitrary Euclidean domain $\Omega$ and with coefficients in the Sobolev space $W^{m, \infty}(\Omega)$ then, under some additional technical assumptions, it has a fundamental solution $K(t, x, y)$ that satisfies

$$
|K(t, x, y)| \leq c_{\epsilon, \beta} t^{-N / 2 m} \exp \left\{-\left(\sigma_{m}-\epsilon\right) \frac{d_{\beta}(x, y)^{2 m /(2 m-1)}}{t^{1 /(2 m-1)}}+c^{\prime} t\right\}
$$

for all $\epsilon, \beta>0$ and all $x, y \in \Omega, t>0$. Here $\left\{d_{\beta}(\cdot, \cdot)\right\}$ is a family of Finsler-type metrics depending on a large parameter $\beta$ and defined by

$$
d_{\beta}(x, y)=\sup \left\{\phi(y)-\phi(x) \mid \phi \in \mathcal{E}_{\beta}\right\}
$$

where

$$
\mathcal{E}_{\beta}=\left\{\phi \in C^{m}(\Omega), a(z, \nabla \phi(z)) \leq 1,\left|D^{\alpha} \phi\right| \leq \beta^{|x|}, 2 \leq|\alpha| \leq m\right\}
$$

It is immediate that $d(x, y) \geq d_{\beta}(x, y)$ for all $\beta>0$ and all $x, y \in \Omega$. The conjecture was put forward in [5] that

$$
\frac{d_{\beta}(x, y)}{d(x, y)} \rightarrow 1, \quad \text { as } \beta \rightarrow \infty
$$

uniformly in $x$ and $y$, provided some regularity is imposed on the boundary. This would allow one to improve the sharpness of (4) by replacing $d_{\beta}(x, y)$ by $d(x, y)$. For 
$\Omega=\mathbf{R}^{N}$ this would agree with the asymptotics of [18] and no further improvement would be possible other than weakening the regularity assumptions. Modulo the regularity condition, this would therefore be the higher order analogue of a well known result of Davies [9].

A proof of the above conjecture is a corollary of our main theorem, Theorem 2. However, for the sake of greater generality as well as greater clarity, we prefer to work in the more abstract context of a general Finsler metric on a Euclidean domain $\Omega$. The results obtained depend on two regularity assumptions on the metric, hypotheses (H1) and (H2). Examples are given that provide sufficient conditions for them to be valid. In the preliminaries section we have also included the necessary background material so as to make the paper self-contained and available to those unfamiliar with Finsler metrics. Finally, we mention that (5) has already been proved in [15] for the special case where $H=(-\Delta)^{m}$ and $\Omega$ is a bounded domain with $C^{2}$ boundary.

Acknowledgements. I thank E. B. Davies for several useful discussions and Z. Shen and Eu. Shargorodsky for helpful comments. The work was carried out with EPSRC support under grant number GR/K00967.

\section{Preliminaries and examples}

Notation. Given a multi-index $\alpha=\left(\alpha_{1}, \ldots, \alpha_{N}\right)$ we write $|\alpha|:=\alpha_{1}+\cdots+\alpha_{N}$ and $\alpha !=\alpha_{1} ! \ldots \alpha_{N} !$; we use the standard notation $D^{\alpha}$ for the differential operator $\left(\partial / \partial x_{1}\right)^{\alpha_{1}} \ldots\left(\partial / \partial x_{N}\right)^{\alpha_{N}}$. For any positive integer $r$ we denote by $C_{b}^{r}(\Omega)$ the space of all functions on $\Omega$ all of whose derivatives of order up to $r$ are continuous and bounded on $\Omega$. The symbol $c$ will denote a constant whose value may change from line to line.

A Finsler metric on a manifold is the assignment of a length to tangent vectors. Unlike the Riemannian case, this length is given by a norm - not an inner product. Let $\Omega$ be a domain in $\mathbf{R}^{N}$ with (possibly empty) boundary $\partial \Omega$.

Definition 1. A Finsler metric on $\Omega$ is a map

$$
p: \Omega \times \mathbf{R}^{N} \rightarrow \mathbf{R}
$$

such that

(i) For any $(x, \xi) \in \Omega \times \mathbf{R}^{N}$ we have $p(x, \xi) \geq 0$, with equality holding iff $\xi=0$;

(ii) $p(x, \lambda \xi)=|\lambda| p(x, \xi), \quad(x, \xi) \in \Omega \times \mathbf{R}^{N}, \lambda \in \mathbf{R}$;

(iii) For $x \in \Omega$ there exists $s(x)>0$ such that $\left\{g_{i j}\right\}:=\left\{\frac{1}{2} \frac{\partial^{2} p^{2}}{\partial \xi_{i} \partial \xi_{j}}\right\} \geq s(x) I$ in the sense of matrices.

The above conditions imply $[17, \mathrm{p} .7]$ that $p(x, \xi)$ is convex in $\xi$, so that $\xi \mapsto p(x, \xi)$ is a norm for each $x \in \Omega$. 
Definition 2. We say that the Finsler metric $p(\cdot, \cdot)$ belongs to the class $\mathcal{F}_{m}(\Omega)$, $m \in \mathbf{Z}_{+}$, if

(i) there exists a constant $c>1$ such that

$$
c^{-1}|\xi| \leq p(x, \xi) \leq c|\xi|, \quad \text { all } x \in \Omega, \xi \in \mathbf{R}^{N}
$$

(ii) there exists a constant $c$ such that for any two multi-indices $\alpha, \beta$ with $1 \leq|\alpha|+|\beta| \leq m+1$ we have

$$
\left|D_{x}^{\alpha} D_{\xi}^{\beta} p(x, \xi)\right| \leq c|\xi|^{1-|\beta|} \quad \text { all } x \in \Omega, \xi \in \mathbf{R}^{N} \backslash\{0\} ;
$$

(iii) the constant $s(x)$ in (iii) above can be taken to be independent of $x \in \Omega$.

A Finsler metric $p \in \mathcal{F}_{m}(\Omega)$ induces a distance $d(\cdot, \cdot)$ on $\Omega$ as follows: Given an absolutely continuous path $\gamma(t), t \in[0,1]$, contained in $\Omega$, we define its length $l(\gamma)$ by

$$
l(\gamma)=\int_{0}^{1} p(\gamma(t), \dot{\gamma}(t)) d t .
$$

The distance of two points $x$ and $y$ is then defined to be

$$
d(x, y)=\inf _{\gamma} l(\gamma)
$$

where the infimum is taken over the set of all absolutely continuous paths $\gamma$ in $\Omega$ that join the points $x$ and $y$. This defines a distance on $\Omega$, with length element $d s=p(x, d x)$. It is an immediate consequence of part (i) of Definition 2 that

$$
c^{-1} d_{E u c}(x, y) \leq d(x, y) \leq c d_{E u c}(x, y), \quad x, y \in \Omega,
$$

where $d_{E u c}(x, y)$ denotes the distance on $\Omega$ induced by the Euclidean metric, i.e. by the metric $p_{\text {Euc }}(x, \xi)=|\xi|$.

Of course, a Finsler metric of the form $p(x, \xi)=\left(\sum_{i, j=1}^{N} a_{i j}(x) \xi_{i} \xi_{j}\right)^{1 / 2}$ is also a Riemannian metric. Going back to the connection between Finsler metrics and elliptic operators, we point out that Riemannian metrics correspond not only to second order operators but also to those higher order operators whose principal symbol is an integer power of the symbol of a second order operator. This is an immediate consequence of Proposition 1 below.

Given a Finsler metric $p(\cdot, \cdot)$ we define

$$
p_{*}(x, \xi)=\sup _{\eta \neq 0} \frac{\xi \cdot \eta}{p(x, \eta)}, \quad x \in \Omega, \xi \in \mathbf{R}^{N} .
$$


Conditions (i)-(iii) of Definition 1 then imply that $p_{*}(\cdot, \cdot)$ is also a Finsler metric with $[17$, p. 22]

$$
\frac{1}{2} \frac{\partial^{2} p_{*}^{2}}{\partial \xi_{i} \partial \xi_{j}}=g^{i j}(x, \xi)
$$

where $\left\{g^{i j}(x, \xi)\right\}$ is the inverse matrix of $\left\{g_{i j}(x, \xi)\right\}$. It follows from (8) that any differentiability properties of $p(\cdot, \cdot)$ are also valid for $p_{*}(x, \xi)$ and, in particular, that $p(\cdot, \cdot)$ belongs to $\mathcal{F}_{m}(\Omega)$ if and only if $p_{*}(\cdot, \cdot)$ does. We also note the reflexivity relation, $p_{* *}=p$.

We next define the class of elliptic operators to which our main theorem will be applied.

Definition 3. Let $H$ be a uniformly elliptic, self-adjoint operator of the form (1), satisfying Dirichlet boundary conditions on $\partial \Omega$. We say that $H$ belongs to the class $\mathcal{D}_{m}(\Omega)$ if

(i) the coefficients $a_{x \beta}(x)$ are real and belong to $C_{b}^{m+1}(\Omega)$;

(ii) $\langle H f, f\rangle \geq C\left\|(-\Delta)^{m} f\right\|_{2}^{2}, \forall f \in C_{c}^{\infty}(\Omega)$;

(iii) the relation $p_{*}(x, \xi)=a(x, \xi)^{1 / 2 m}$ defines a Finsler metric $p \in \mathcal{F}_{m}(\Omega)$;

(iv) the principal symbol of $H$ is strongly convex ${ }^{l}$.

Example. Consider the operator $H_{1}$ on $\Omega \subset \mathbf{R}^{2}$ with symbol $a(x, \xi)=a_{0} \xi_{1}^{4}+$ $4 a_{1} \xi_{1}^{3} \xi_{2}+6 a_{2} \xi_{1}^{2} \xi_{2}^{2}+4 a_{3} \xi_{1} \xi_{2}^{3}+a_{4} \xi_{2}^{4}$, where $a_{i}$ are functions in $C_{b}^{3}(\Omega)$. It can be seen that (iii) is satisfied if and only if the values of the polynomial $\left(a_{0} a_{2}-a_{1}^{2}\right) u^{4}+$ $2\left(a_{0} a_{3}-a_{1} a_{2}\right) u^{3}+\left(a_{0} a_{4}+2 a_{1} a_{3}-3 a_{2}^{2}\right) u^{2}+2\left(a_{1} a_{4}-a_{2} a_{3}\right) u+\left(a_{2} a_{4}-a_{3}^{2}\right)$ are bounded away from zero. Moreover, (iv) is satisfied if and only if the matrix

$$
A_{1}(x):=\left(\begin{array}{lll}
a_{0} & a_{1} & a_{2} \\
a_{1} & a_{2} & a_{3} \\
a_{2} & a_{3} & a_{4}
\end{array}\right)
$$

is positive semi-definite for all $x \in \Omega$.

Example. Let $H_{2}$ on $\Omega \subset \mathbf{R}^{2}$ have symbol $\sum_{r=6}^{6}\left(\begin{array}{l}6 \\ r\end{array}\right) a_{r}(x) \xi_{1}^{6-r} \xi_{2}^{r}$, where $a_{i} \in C_{b}^{4}(\Omega)$. It is not difficult to see that $H \in \mathcal{D}_{3}(\Omega)$ if and only if (i) values of the polynomial $p(u)=$ $\left(a_{0} a_{2}-a_{1}^{2}\right) u^{8}+4\left(a_{0} a_{3}-a_{1} a_{2}\right) u^{7}+2\left(3 a_{0} a_{4}+2 a_{1} a_{3}-5 a_{2}^{2}\right) u^{6}+4\left(a_{0} a_{5}+4 a_{1} a_{4}-5 a_{2} a_{3}\right) u^{5}+$ $\left(a_{0} a_{6}+14 a_{1} a_{5}+5 a_{2} a_{4}-20 a_{3}^{2}\right) u^{4}+4\left(a_{1} a_{6}+4 a_{2} a_{5}-5 a_{3} a_{4}\right) u^{3}+2\left(3 a_{2} a_{6}+2 a_{3} a_{5}-5 a_{4}^{2}\right) u^{2}+$ $4\left(a_{3} a_{6}-a_{4} a_{5}\right) u+\left(a_{4} a_{6}-a_{5}^{2}\right)$ are bounded away from zero and (ii) the matrix

\footnotetext{
${ }^{1}$ The symbol $a(x, \xi)$ is strongly convex if it has the form $a(x, \xi)=\sum_{\mid m=2 m} c_{r}^{2 m} a_{\gamma}(x) \xi^{\prime}$, where $\left\{a_{\alpha+\beta}\right\}_{|x|=\mid \xi ;=m}$ is a positive semi-definite matrix. Here $c_{\gamma}^{2 m}=(2 m) ! / \gamma !$.
} 


$$
A_{2}(x):=\left(\begin{array}{cccc}
a_{0} & a_{1} & a_{2} & a_{3} \\
a_{1} & a_{2} & a_{3} & a_{4} \\
a_{2} & a_{3} & a_{4} & a_{5} \\
a_{3} & a_{4} & a_{5} & a_{6}
\end{array}\right)
$$

is positive semi-definite for all $x \in \Omega$.

We shall need the following result:

Proposition 1. (a) For fixed $y \in \Omega$ the function $x \mapsto d(x, y)$ is Lipschitz continuous and we have

$$
p_{*}\left(x, \nabla_{x} d(x, y)\right) \leq 1
$$

at all points $x \in \Omega$ where $\nabla_{x} d(x, y)$ is defined.

(b) We have

$$
d(x, y)=\sup \{\phi(y)-\phi(x) \mid \phi \in \mathcal{E}\}
$$

where $\mathcal{E}=\left\{\phi \mid \phi\right.$ is Lipschitz continuous on $\Omega$ and $p_{*}(z, \nabla \phi(z)) \leq 1$ a.e. $\left.z \in \Omega\right\}$.

Proof. This is Lemma 1.3 of [2].

A (parametrized) path $\gamma(t)$ in $\Omega$ is called a geodesic if it locally realizes the infimum in (6), that is if given any point $x_{0}=\gamma\left(t_{0}\right)$ on the path, there exists $\epsilon>0$ such that for any point $x=\gamma(t)$ that satisfies $d\left(x_{0}, x\right)<\epsilon$ we have $d\left(x_{0}, x\right)=\int_{t_{0}}^{t} p(\gamma(s), \dot{\gamma}(s)) d s$. Geodesics satisfy the geodesic equation:

$$
\ddot{\gamma}_{k}+\sum_{i, j=1}^{N} \Gamma_{i j}^{k}(\gamma, \dot{\gamma}) \dot{\gamma}_{i} \dot{\gamma}_{j}=0, \quad k=1, \ldots, N .
$$

The functions $\Gamma_{i j}^{k}(x, \xi)$ are induced by the $g_{i j}$ 's in the same way (formally) as in the Riemannian case. Note that while in the Riemannian case they only depend on $x$, in general they depend on the full line element $(x, \xi)$.

Given $x \in \Omega$ there exists $\epsilon>0$ small enough such that for any $\eta \in \mathbf{R}^{N},|\eta|=1$, the geodesic equation ( 9 ) subject to the initial conditions

$$
\gamma(0)=x, \quad \dot{\gamma}(0)=\eta
$$

has a unique solution on the interval $[0, \epsilon]$. Let us denote that solution by $\gamma_{x, \eta}(t)$. The exponential map $\exp _{x}$ is defined for $|\xi|<\epsilon$ by 
It clearly satisfies

$$
\left\{\begin{array}{l}
\exp _{x} \xi=\gamma_{x, \xi /|\xi|}(|\xi|), \quad \xi \neq 0 \\
\exp _{x} 0=x
\end{array}\right.
$$

$$
d\left(x, \exp _{x} \xi\right)=p(x, \xi), \quad \text { all }|\xi|<\epsilon .
$$

The assumption $p(\cdot, \cdot) \in \mathcal{F}_{m}(\Omega)$ together with theorems on the dependence of the solution of initial value problems on the initial data [7, p. 131] implies that $\exp _{x}$ is a $C^{m}$-diffeomorphism of the set $\left\{\xi \in \mathbf{R}^{N}|0<| \xi \mid<\epsilon\right\}$ onto its range, which is a neighbourhood of $x$ minus $\{x\}$. (By $C^{m}$-diffeomorphism we mean a map that is 1-1, onto, of class $C^{m}$, whose inverse is also $C^{m}$.) At the point $\xi=0$ it is not $C^{m}$ but only Lipschitz continuous, due to the singularity of the functions $\left\{\Gamma_{i j}^{k}\right\}$ at that point. For detailed proof of the above statements we refer to Section 1.6.2 of [1].

We introduce two basic hypotheses on $p(\cdot, \cdot)$.

Hypothesis (H1). There exists a domain $\Omega^{\prime}$ with $\Omega^{\prime} \supset \supset \Omega$ and such that $p(\cdot, \cdot)$ extends to a Finsler metric $p^{\prime}(\cdot, \cdot) \in \mathcal{F}_{m}\left(\Omega^{\prime}\right)$.

Hence we have two distances: the original distance $d(x, y)$ on $\Omega$ and the distance $d^{\prime}(x, y)$ on $\Omega^{\prime}$; their restrictions on $\Omega$ of course do not coincide. We shall omit the primes however from $p^{\prime}(x, \xi)$ and $p_{*}^{\prime}(x, \xi)$ and write $p(x, \xi)$ and $p_{*}(x, \xi)$ instead. Of course, (H1) may well be satisfied without any regularity assumptions on $\partial \Omega$. The next example provides a sufficient condition which depends on boundary regularity.

Example. Let $\Omega$ be a bounded domain with $C^{m+1}$ boundary and assume that $p(\cdot, \cdot)$ lies in $\mathcal{F}_{m}(\Omega)$. Assume that for each $\xi \in \mathbf{R}^{N}$ the function $p_{\xi}(x):=p(x, \xi), x \in \Omega$, belongs to $C^{m+1}(\bar{\Omega})$. By [12, Ch. 5, Prop. 4.8] there exists a linear extension map $E$ that extends each function $f \in C^{m+1}(\bar{\Omega})$ to a function $E f=: \tilde{f}$ on a neighbourhood $\Omega^{\prime}$ of $\Omega$, and such that $\tilde{f} \in C^{m+1}\left(\Omega^{\prime}\right)$. We claim that $\tilde{p}(x, \xi):=\tilde{p}_{\xi}(x)$ is then a Finsler metric in $\mathcal{F}_{m}\left(\Omega^{\prime}\right)$. Indeed, we have

$$
\tilde{p}(x, \lambda \xi)=\left(E p_{\lambda \xi}\right)(x)=E\left(|\lambda| p_{\xi}\right)(x)=|\lambda| \tilde{p}(x, \xi) .
$$

Moreover, the validity of (i)-(iii) of Definition 2 on $\bar{\Omega}$ combined with the fact that $\tilde{p}$ lies in $C^{m+1}\left(\Omega^{\prime}\right)$ implies that those properties are also valid on $\Omega^{\prime}$, by making $\Omega^{\prime}$ smaller if necessary. Hence $\tilde{p} \in \mathcal{F}_{m}\left(\Omega^{\prime}\right)$ and $(\mathrm{H} 1)$ is satisfied.

It follows from ( $\mathrm{H} 1)$ that there exists $\delta_{1}>0$ small enough so that the neighbourhoods

$$
\Omega_{\delta}:=\left\{x \in \Omega^{\prime} \mid d^{\prime}(x, \Omega)<\delta\right\}, \quad 0<\delta \leq \delta_{1},
$$

are compactly contained in $\Omega^{\prime}$. On each $\Omega_{\delta}, 0<\delta \leq \delta_{1}$, we let $d^{(\delta)}(x, y)$ denote the 
Finsler distance induced by $p(x, \xi)$. Similarly to (7), there exist positive constants $k_{1}, k_{2}$ such that

$$
k_{1} d_{E u c}^{(\delta)}(x, y) \leq d^{(\delta)}(x, y) \leq k_{2} d_{E u c}^{(\delta)}(x, y), \quad 0<\delta \leq \delta_{1}, \quad x, y \in \Omega_{\delta}
$$

(Here we have denoted by $d_{E u c}^{(\delta)}(x, y)$ the Euclidean geodesic distance on $\Omega_{\delta}$.) The constants $k_{1}, k_{2}$ in (12) can be taken to be independent of $\delta$ since they can only become better as the domains $\Omega_{\delta}$ shrink to $\Omega$. To simplify our notation and without any loss of generality we assume that $k_{2}=1$ so that

$$
k_{1} d_{E u c}^{(\delta)}(x, y) \leq d^{(\delta)}(x, y) \leq d_{E u c}^{(\delta)}(x, y), \quad 0<\delta \leq \delta_{1}, \quad x, y \in \Omega_{\delta}
$$

We state a second hypothesis:

Hypothesis (H2). There exists $v \in(0,1]$ and $\delta_{1}^{\prime} \leq \delta_{1}$ such that

$$
\left(1-c \delta^{v}\right) d(x, y) \leq d^{(\delta)}(x, y) \leq d(x, y)
$$

for all $0<\delta \leq \delta_{1}^{\prime}$ and all $x, y \in \Omega$.

By making $\delta_{1}$ smaller if necessary, we may assume that $\delta_{1}^{\prime}=\delta_{1}$, and we do so.

Examples. (A) If $\Omega$ is geodesically convex, i.e. if any two points can be connected by a geodesic, then $(\mathrm{H} 2)$ is valid with $v=1$.

(B) If $\Omega$ is bounded and star-shaped, around the origin, say, then (H2) is valid with $v=1$. This is seen as follows: given a path $\gamma$ in $\Omega_{\delta}$, define a path $\gamma_{1}$ in $\Omega$ by $\gamma_{1}=(1+\delta)^{-1} \gamma$. Then $p\left(\gamma_{1}, \dot{\gamma}_{1}\right)=(1+\delta)^{-1} p\left(\gamma_{1}, \dot{\gamma}\right)$. Moreover

$$
\left|p\left(\gamma_{1}, \dot{\gamma}\right)-p(\gamma, \dot{\gamma})\right| \leq c\left|\gamma-\gamma_{1}\right| p(\gamma, \dot{\gamma}) \leq c \delta p(\gamma, \dot{\gamma})
$$

for $\delta$ small enough. Combining the above we obtain $p\left(\gamma_{1}, \dot{\gamma}_{1}\right) \leq\left(1+c^{\prime} \delta\right) p(\gamma, \dot{\gamma})$. This implies (H2) with $v=1$.

(C) If the Finsler metric is Riemannian and $\Omega$ has a boundary which is uniformly $C^{2}$ then (H2) is valid with $v=1$. This has been shown in [15] for the Euclidean metric; with minor modifications the proof carries through in the general case: given a path $\gamma$ contained in $\Omega_{\delta}$ one constructs a path $\gamma_{1}$ contained in $\bar{\Omega}$ and given by $\gamma_{1}(t)=\tau(\gamma(t))$, where $\tau x$ is equal to $x$ when $x \in \Omega$ and equal to the orthogonal projection of $x$ onto $\partial \Omega$ when $x \in \Omega_{z} \backslash \Omega$. Then one compares the lengths of the two paths. The argument does not hold for a general Finsler metric, despite the underlying Euclidean structure. The point, of course, is that a (Euclidean) orthogonal projection $P \xi$ of a tangent vector $\xi$ does not necessarily satisfy $p(x, P \xi) \leq p(x, \xi)$.

(D) Suppose $\Omega$ is bounded with $C^{i}$ boundary. Then (H2) is valid with $v=1 / 2$. This is proved in Proposition 8. 


\section{Main estimates}

We fix a positive integer $m \geq 2$ and a Finsler metric $p \in \mathcal{F}_{m}(\Omega)$. We assume that (H1) and (H2) are satisfied. Given $\beta>1$ we define the set

$$
\mathcal{E}_{\beta}=\left\{\phi \in C^{m}(\Omega) \mid p_{*}(x, \nabla \phi(x)) \leq 1 \text { and }\left|D^{\alpha} \phi(x)\right| \leq \beta^{|x|}, x \in \Omega, 2 \leq|\alpha| \leq m\right\}
$$

and the Finsler-type distance

$$
d_{\beta}(x, y)=\sup \left\{\phi(y)-\phi(x) \mid \phi \in \mathcal{E}_{\beta}\right\}
$$

It is immediate from Proposition 1 that $d_{\beta}(x, y) \leq d(x, y)$; our aim is to prove the following

Theorem 2. Assume that $p \in \mathcal{F}_{m}(\Omega)$ and that $(\mathrm{H} 1)$ and $(\mathrm{H} 2)$ are valid. Then there exist constants $c, \beta_{0}>0$ such that for $\beta \geq \beta_{0}$ we have

for all $x, y \in \Omega$.

$$
\left(1-c \beta^{-v /(v+1)}\right) d(x, y) \leq d_{\beta}(x, y) \leq d(x, y),
$$

The theorem has an application in the theory of higher order elliptic operators. The estimate in the following corollary is sharp, as can be seen by comparing with the short-time asymptotics of [18] which, however, are only valid for $\Omega=\mathbf{R}^{N}$ and for $x$ and $y$ sufficiently close. It improves upon the estimates of [5], subject however to stronger hypotheses. Let $\sigma_{m}=(2 m-1)(2 m)^{-2 m /(2 m-1)} \sin (\pi / 4 m-2)$.

Corollary 3. Let $H$ be an operator in $\mathcal{D}_{m}(\Omega), 2 m>N$, and assume that the induced Finsler metric $p(\cdot, \cdot)$ satisfies $(\mathrm{H} 1)$ and $(\mathrm{H} 2)$. Then given $\epsilon>0$ there exists $c_{\mathrm{c}}<\infty$ such that the fundamental solution $K(t, x, y)$ of the parabolic equation $\partial u_{t} / \partial t=-H u_{t}$ satisfies

$$
|K(t, x, y)| \leq c_{\epsilon} t^{-N / 2 m} \exp \left\{-\left(\sigma_{m}-\epsilon\right) \frac{d(x, y)^{2 m /(2 m-1)}}{t^{1 /(2 m-1)}}+c^{\prime} t\right\}
$$

for all $t>0$ and $x, y \in \Omega$.

Proof. Let $\epsilon>0$ be given. Corollary 9 of [5] states that given $\eta>0$ (small) and $\beta>0$ (large) there exists a constant $c_{\eta, \beta}$ such that

$$
|K(t, x, y)| \leq c_{\eta, \beta} t^{-N / 2 m} \exp \left\{-\left(\sigma_{m}-\eta\right) \frac{d_{\beta}(x, y)^{2 m /(2 m-1)}}{t^{1 /(2 m-1)}}+c^{\prime} t\right\}
$$

for all $t>0$ and $x, y \in \Omega$. It follows from Theorem 2 that we can find $\eta>0$ small enough and $\beta$ large enough so that $\left(\sigma_{m}-\eta\right) d_{\beta}(x, y) \geq\left(\sigma_{m}-\epsilon\right) d(x, y)$ for all $x, y \in \Omega$. The result then follows. 
Remark. It is not possible to remove the $\epsilon$ in (16), as it compensates for subexponential terms that we have omitted from the RHS of (16). See [18].

Let $B(r)$ denote the open Euclidean ball of radius $r$ and centered at the origin. Let $k(z)$ be a non-negative smooth function with support in $B(1)$ and such that $\int k(z) d z=1$. For $0<\delta<\delta_{1}$, where $\delta_{1}$ is as in (H1) and $(\mathrm{H} 2)$, let

$$
k_{\delta}(z)=\delta^{-N} k(z / \delta)
$$

Given $y \in \Omega$ and $z \in B(\delta)$, we note from (13) that the Euclidean ball with centre $y \in \Omega$ and radius $|z|$ is compactly contained in $\Omega^{\prime}$ for $|z|$ smaller than a certain $\delta_{1}^{\prime \prime}$, which, for the second and last time, we may assume is $\delta_{1}$ itself by making $\delta_{1}$ smaller if necessary. Hence $d_{E u c}^{\prime}(y, y+z)=|z|$ and therefore, by (13),

$$
d^{\prime}(y, y+z) \leq d_{E u c}^{\prime}(y, y+z)=|z| \leq \delta,
$$

which implies $y+z \in \Omega_{\delta}$.

Hence given a fixed point $x_{0} \in \Omega$ and $0<\delta<\delta_{1} / 2$, the functions

$$
\phi_{\delta}(y)=\int_{B(\delta)} d^{(2 \delta)}\left(x_{0}, y+z\right) k_{\delta}(z) d z, \quad y \in \Omega,
$$

are well defined and smooth on $\Omega$.

Lemma 4. There exist constants $c, c_{1}<\infty$ such that for $0<\delta<\delta_{1} / 2$

(i) $p_{*}\left(y, \nabla \phi_{\delta}(y)\right) \leq 1+c_{1} \delta, \quad$ all $y \in \Omega$;

(ii) $\left|D^{\gamma} \phi_{\delta}(y)\right| \leq c \delta^{-|y|+1}$, all $2 \leq|\gamma| \leq m, y \in \Omega$.

Proof. Let $y \in \Omega$ and $e \in \mathbf{R}^{N},|e|=1$, be fixed and let $r>0$ be sufficiently small so that $y+z+r e \in \Omega_{2 \delta}$ for all $z \in B(\delta)$. Using the triangle inequality for $d^{(2 \delta)}(\cdot, \cdot)$ we have

$$
\begin{aligned}
\phi_{\delta}(y+r e)-\dot{\phi}_{\delta}(y) & =\int_{B(\delta)}\left[d^{(2 \delta)}\left(x_{0}, y+r e+z\right)-d^{(2 \delta)}\left(x_{0}, y+z\right)\right] k_{\delta}(z) d z \\
& \leq \int_{B(\delta)} d^{(2 \delta)}(y+z, y+z+r e) k_{\delta}(z) d z \\
& \leq \int_{B(\delta)} \int_{0}^{1} p(y+z+\text { tre }, r e) k_{\delta}(z) d t d z
\end{aligned}
$$

Dividing both sides by $r$, using the homogeneity of $p$ in the second variable and letting $r \rightarrow 0$ we conclude that 


$$
\begin{aligned}
\nabla \phi_{\delta}(y) \cdot e & \leq \int_{B(\delta)} \int_{0}^{1} p(y+z, e) k_{\delta}(z) d t d z \\
& =\int_{B(\delta)} p(y+z, e) k_{\delta}(z) d z
\end{aligned}
$$

Since

$$
\begin{aligned}
\left|p(y, e)-\int_{B(\delta)} p(y+z, e) k_{\delta}(z) d z\right| & \leq \int_{B(\delta)}|p(y, e)-p(y+z, e)| k_{\delta}(z) d z \\
& \leq \sup _{|z|<\delta}|p(y, e)-p(y+z, e)| \\
& \leq c \delta
\end{aligned}
$$

we conclude that

$$
\nabla \phi_{\delta}(y) \cdot e \leq p(y, e)+c \delta
$$

where the constant $c$ is independent of both $y$ and $\delta$, as well as the point $x_{0}$ used in the definition of the function $\phi_{\delta}$. Therefore

$$
\begin{aligned}
\frac{\nabla \phi_{\delta}(y) \cdot e}{p(y, e)} & \leq 1+\frac{c \delta}{p(y, e)} \\
& \leq 1+c_{1} \delta .
\end{aligned}
$$

Taking the supremum of both sides over all $e \in S^{N-1}$ we conclude that

$$
p_{*}\left(y, \nabla \phi_{\delta}(y)\right) \leq 1+c_{1} \delta, \quad y \in \Omega
$$

Now, let $\alpha$ be any multi-index with $0 \leq|\alpha| \leq m-1$ and let $h$ be a vector in $\mathbf{R}^{N}$ of small length. For $y \in \Omega$ we have

$$
\begin{aligned}
\left|D^{\alpha} \phi_{\delta}(y+h)-D^{\alpha} \phi_{\delta}(y)\right| & \leq \int_{B(\delta)}\left|d^{(2 \delta)}\left(x_{0}, z+y+h\right)-d^{(2 \delta)}\left(x_{0}, z+y\right)\right|\left|D^{\alpha} k_{\delta}(z)\right| d z \\
& \leq \int_{B(\delta)} d^{(2 \delta)}(z+y, z+y+h)\left|D^{\alpha} k_{\delta}(z)\right| d z .
\end{aligned}
$$

If $|h|$ is small enough we have

$$
d^{(2 \delta)}(x, x+h) \leq c|h|, \quad \text { all } x \in \Omega_{\delta_{1}},
$$

where the constant $c$ is independent of $x \in \Omega_{\delta_{1}}$ and $h \in \mathbf{R}^{N}$ (with $|h|$ small). Letting $|h| \rightarrow 0$ we conclude that 


$$
\begin{aligned}
\limsup _{|h| \rightarrow 0} \frac{\left|D^{\alpha} \phi_{\delta}(y+h)-D^{\alpha} \phi_{\delta}(y)\right|}{|h|} & \leq c \int_{B(\delta)}\left|D^{\alpha} k_{\delta}(z)\right| d z \\
& =c \delta^{-|\alpha|} .
\end{aligned}
$$

It follows that if $\gamma$ is a multi-index with $|\gamma|=|\alpha|+1$, then $\left|D^{\gamma} \phi_{\delta}(y)\right| \leq c \delta^{-|\gamma|+1}$ as required.

Lemma 5. There exists $\beta_{1}>0$ and a constant $c>0$ such that

$$
d_{\beta}(x, y) \geq\left(1-c \beta^{-1}\right) d^{\left(2 \beta^{-1}\right)}(x, y)-c \beta^{-1} .
$$

for all $\beta \geq \beta_{1}$ and all $x, y \in \Omega$.

Proof. Let the family $\left\{\phi_{\delta}\right\}$ be as above and define

$$
\tilde{\phi}_{\beta}(y)=\left(1+c_{1} \beta^{-1}\right)^{-1} \phi_{\beta^{-1}}(y), \quad y \in \Omega,
$$

where $c_{1}$ is as in (18). Then

$$
p\left(y, \nabla \tilde{\phi}_{\beta}(y)\right) \leq 1, \quad y \in \Omega
$$

and for any multi-index $\alpha$ with $2 \leq|\alpha| \leq m$ we have

$$
\left|D^{\alpha} \tilde{\phi}_{\beta}(y)\right| \leq \frac{c \beta^{|\alpha|-1}}{1+c_{1} \beta^{-1}}, \quad y \in \Omega,
$$

for all $\beta$ large and all $y \in \Omega$. It follows from (21) and (22) that $\tilde{\phi}_{\beta} \in \mathcal{E}_{\beta}$ provided $\beta$ is large enough.

We now estimate the difference $\tilde{\phi}_{\beta}(y)-\tilde{\phi}_{\beta}(x)$ for given and fixed $x, y \in \Omega$. We take the point $x_{0}$ from which distances are measured, in (17), to be the point $x$. Using the triangle inequality we have

$$
\begin{aligned}
\phi_{\beta^{-1}}(y)-\phi_{\beta^{-1}}(x) & =\int_{B(1 /(\beta))}\left[d^{\left(2 \beta^{-1}\right)}(x, y+z)-d^{\left(2 \beta^{-1}\right)}(x, x+z)\right] k_{1 /(\beta)}(z) d z \\
& \geq \int_{B(1 /(\beta))}\left[d^{\left(2 \beta^{-1}\right)}(x, y)-d^{\left(2 \beta^{-1}\right)}(y, y+z)-d^{\left(2 \beta^{-1}\right)}(x, x+z)\right] k_{1 /(\beta)}(z) d z \\
& \geq d^{\left(2 \beta^{-1}\right)}(x, y)-2 \sup _{u \in \Omega} \sup _{|z| \leq 1 /(\beta)} d^{\left(2 \beta^{-1}\right)}(u, u+z) \\
& \geq d^{\left(2 \beta^{-1}\right)}(x, y)-c \beta^{-1},
\end{aligned}
$$

provided $\beta$ is large enough. We conclude that 


$$
\begin{aligned}
d_{\beta}(x, y) & \geq \tilde{\phi}_{\beta}(y)-\tilde{\phi}_{\beta}(x) \\
& =\left(1+c \beta^{-1}\right)^{-1}\left[\phi_{\beta}(y)-\phi_{\beta}(x)\right] \\
& \geq \frac{d^{\left(2 \beta^{-1}\right)}(x, y)-c \beta^{-1}}{1+c_{1} \beta^{-1}} \\
& \geq d^{\left(2 \beta^{-1}\right)}(x, y)-c d^{\left(2 \beta^{-1}\right)}(x, y) \beta^{-1}-c \beta^{-1},
\end{aligned}
$$

as required.

Lemma 6. (i) For every $x, y \in \Omega_{\delta_{1} / 2}$ with $d^{\left(\delta_{1}\right)}(x, y)<\delta_{1} / 2$ there exists a geodesic $\gamma$ joining $x$ and $y$, contained in $\Omega_{\delta_{1}}$, and such that $l(\gamma)=d^{\left(\delta_{1}\right)}(x, y)$.

(ii) For fixed $x \in \Omega_{\delta_{1} / 2}$ the function $y \mapsto d^{\left(\delta_{1}\right)}(x, y)$ is $C^{m}$ when restricted on the set $\left\{y \in \Omega_{\delta_{1}} \mid 0<d^{\left(\delta_{1}\right)}(x, y)<\delta_{1} / 2\right\}$ and, further, there exists a constant $M$ such that

$$
\left|D_{y}^{\alpha} d^{\left(\delta_{1}\right)}(x, y)\right| \leq M
$$

for all $x \in \Omega_{\delta_{1} / 2}$, all $y$ such that $\delta_{1} / 10 \leq d^{\left(\delta_{1}\right)}(x, y) \leq \delta_{1} / 3$ and all $2 \leq|\alpha| \leq m$.

Proof. It follows from the Hopf-Rinow theorem for Finsler spaces [1, Theorem 1.6.9] that any geodesic in $\Omega_{\delta_{1}}$ can be extended until it "hits" $\partial \Omega_{\delta_{1}}$. Hence for $x \in \Omega_{\delta_{1} / 2}$, $\exp _{x} \xi$ is defined (and lies in $\Omega_{\delta_{1}}$ ) for all $\xi$ such that $p(x, \xi)<\delta_{1} / 2$ (recall (11)). From our earlier remarks on the exponential map we conclude that

$$
\exp _{x}:\left\{\xi \in \mathbf{R}^{N} \mid 0<p(x, \xi)<\delta_{1} / 2\right\} \rightarrow\left\{y \in \Omega^{\prime} \mid 0<d^{\left(\delta_{1}\right)}(x, y)<\delta_{1} / 2\right\}
$$

is well defined and is a $C^{m}$-diffeomorphism. This proves (i). Moreover, by (11), we have

$$
d^{\left(\delta_{1}\right)}(x, y)=p\left(x, \exp _{x}^{-1} y\right)
$$

for all $y$ with $0<d^{\left(\delta_{1}\right)}(x, y)<\delta_{1} / 2$. By the properties of continuous functions on compact sets and the fact that $p \in \mathcal{F}_{m}\left(\Omega_{\delta_{1}}\right)$ we conclude that there exists a constant $M$ such that

$$
\sup _{\delta_{1} / 10 \leq d^{\left(\delta_{1}\right)}(x, y) \leq \delta_{1} / 3}\left|D_{y}^{\alpha} d^{\left(\delta_{1}\right)}(x, y)\right| \leq M, \quad 2 \leq|\alpha| \leq m
$$

The size of the constant $M$ depends on $L^{\infty}$-estimates of $p(\cdot, \cdot)$ and its derivatives in the $\delta_{1}$-neighbourhood of $x$. The fact that $p(\cdot, \cdot) \in \mathcal{F}_{m}\left(\Omega_{\delta_{1}}\right)$ implies that those estimates are uniform with respect to $x \in \Omega_{\delta_{1} / 2}$ and therefore that $M$ may be taken to be independent of $x \in \Omega_{\delta_{1} / 2}$.

Lemma 7. Given $x_{0} \in \Omega_{\delta_{1} / 2}$ there exists a function $\pi_{x_{0}} \in C_{b}^{m}(\Omega)$ such that 
(i) $p_{*}\left(x, \nabla \pi_{x_{0}}(x)\right) \leq 1, \quad x \in \Omega$;

(ii) $\left|D^{\alpha} \pi_{x_{0}}(x)\right| \leq c, \quad x \in \Omega, 2 \leq|\alpha| \leq m$;

(iii) if $\delta_{1} / 8 \leq d^{\left(\delta_{1}\right)}\left(x_{0}, x\right) \leq \delta_{1} / 4$, then $\pi_{x_{0}}(x)=d^{\left(\delta_{1}\right)}\left(x_{0}, x\right)$;

moreover, the constant $c$ can be taken to be independent of $x_{0} \in \Omega_{\delta_{1} / 2}$.

Proof. Let $\psi: \mathbf{R}_{+} \rightarrow \mathbf{R}$ be a smooth function satisfying

$$
0 \leq \psi^{\prime}(s) \leq 1, \quad \text { all } s \in \mathbf{R}_{+},
$$

as well as

$$
\psi(s)= \begin{cases}(1 / 8)-10^{-2} & \text { if } 0 \leq s \leq 1 / 10 \\ s & \text { if } 1 / 8 \leq s \leq 1 / 4 \\ (1 / 4)+10^{-2} & \text { if } s \geq 1 / 3\end{cases}
$$

Let $\psi_{1}(s)=\delta_{1} \psi\left(\delta_{1}^{-1} s\right)$ so that $0 \leq \psi_{1}^{\prime}(s) \leq 1$ and

$$
\psi_{1}(s)= \begin{cases}\left\{(1 / 8)-10^{-2}\right\} \delta_{1} & \text { if } 0 \leq s \leq \delta_{1} / 10 \\ s & \text { if } \delta_{1} / 8 \leq s \leq \delta_{1} / 4 \\ \left\{(1 / 4)+10^{-2}\right\} \delta_{1} & \text { if } s \geq \delta_{1} / 3\end{cases}
$$

There also exists a positive constant $c$ such that

$$
\left|\psi_{1}^{(k)}(s)\right|<c, \quad \text { all } 2 \leq k \leq m, s \in \mathbf{R}_{+} .
$$

Given $x_{0} \in \Omega_{\delta_{1} / 2}$ let $\pi_{x_{0}}(x)=\psi_{1}\left(d^{\left(\delta_{1}\right)}\left(x_{0}, x\right)\right), x \in \Omega$. Since $\psi_{1}^{\prime}$ vanishes outside the interval $\left[\delta_{1} / 10, \delta_{1} / 3\right]$, we conclude from (25) and (26) that $\psi_{1} \in C_{b}^{m}(\Omega)$ and that there exists a constant $c$ such that $\left|D^{\alpha} \pi_{x_{0}}(x)\right| \leq c$ for all $2 \leq|\alpha| \leq m$ and $x \in \Omega$. Moreover

$$
\nabla \pi_{x_{0}}(x)=\psi_{1}^{\prime}\left(d^{\left(\delta_{1}\right)}\left(x_{0}, x\right)\right) \nabla_{x} d^{\left(\delta_{1}\right)}\left(x_{0}, x\right)
$$

and therefore, by homogeneity and part (a) of Proposition 1,

$$
\begin{aligned}
p_{*}\left(x, \nabla \pi_{x_{0}}(x)\right) & =\psi_{1}^{\prime}\left(d^{\left(\delta_{1}\right)}\left(x_{0}, x\right)\right) p_{*}\left(x, \nabla_{x} d^{\left(\delta_{1}\right)}\left(x_{0}, x\right)\right) \\
& \leq 1
\end{aligned}
$$

for all $x \in \Omega$. Finally, it is immediate that if $\delta_{1} / 8 \leq d^{\left(\delta_{1}\right)}\left(x_{0}, x\right) \leq \delta_{1} / 4$ then $\pi_{x_{0}}(x)=$ $d^{\left(\delta_{1}\right)}\left(x_{0}, x\right)$.

We can now prove the main theorem of the paper. 
Proof of Theorem 2. Let $x, y \in \Omega$ be given and $\eta \in(0,1)$ be fixed. We assume that $\beta$ is at least large enough so that $\beta^{-n}<\delta_{1} / 8$. From Lemma 5 and (H2) we then have that if also $\beta>\beta_{1}$ then

$$
\begin{aligned}
d_{\beta}(x, y) & \geq\left(1-c \beta^{-1}\right) d^{\left(2 \beta^{-1}\right)}(x, y)-c \beta^{-1} \\
& \geq\left(1-c \beta^{-1}\right)\left\{\left(1-c \beta^{-v}\right) d(x, y)\right\}-c \beta^{-1} \\
& \geq\left(1-c^{\prime} \beta^{-\nu}\right) d(x, y)-c \beta^{-1}
\end{aligned}
$$

and therefore

$$
d_{\beta}(x, y) \geq\left(1-c^{\prime} \beta^{-v}-c \beta^{-(1-\eta)}\right) d(x, y), \quad \text { if } d(x, y) \geq \beta^{-\eta} .
$$

Suppose now that $d(x, y) \leq \beta^{-\eta}$. Then $d^{\left(\delta_{1}\right)}(x, y) \leq d(x, y) \leq \beta^{-\eta} \leq \delta_{1} / 8$ and therefore, by (24), there exists $\xi \in \mathbf{R}^{N}$ defined by $\exp _{x} \xi=y$. Let

$$
x_{0}:=\exp _{x}\left\{\frac{\left(\delta_{1} / 4\right) \xi}{p(x, \xi)}\right\}
$$

This is well defined and lies in $\Omega_{\delta_{1} / 2}$, again by (24). Let $\phi(z)=\pi_{x_{0}}(z)$, where $\pi_{x_{0}}$ is as in Lemma 7. From the fact that $d^{\left(\delta_{1}\right)}\left(x_{0}, x\right)=\delta_{1} / 4$ follows that

$$
\phi(x)=d^{\left(\delta_{1}\right)}\left(x_{0}, x\right) .
$$

The fact that $y$ lies on the geodesic with endpoints $x$ and $x_{0}$ implies that

$$
d^{\left(\delta_{1}\right)}\left(x_{0}, y\right)<d^{\left(\delta_{1}\right)}\left(x_{0}, x\right)=\delta_{1} / 4
$$

Since we also have

$$
\begin{aligned}
d^{\left(\delta_{1}\right)}\left(x_{0}, y\right) & \geq d^{\left(\delta_{1}\right)}\left(x_{0}, x\right)-d^{\left(\delta_{1}\right)}(x, y) \\
& \geq \delta_{1} / 4-d(x, y) \\
& \geq \delta_{1} / 8
\end{aligned}
$$

we conclude that

$$
\phi(y)=d^{\left(\delta_{1}\right)}\left(x_{0}, y\right) .
$$

From Lemma 7 it follows that $\phi \in \mathcal{E}_{\beta}$ provided $\beta$ is large enough. Moreover, using (28) and (29), we have for such $\beta$

$$
\begin{aligned}
d_{\beta}(x, y) & \geq \phi(x)-\phi(y) \\
& =d^{\left(\delta_{1}\right)}\left(x_{0}, x\right)-d^{\left(\delta_{1}\right)}\left(x_{0}, y\right) .
\end{aligned}
$$


The fact that $y$ is a geodesic that passes from $x, y$ and $x_{0}$ (in that order) implies that $d^{\left(\delta_{1}\right)}\left(x, x_{0}\right)=d^{\left(\delta_{1}\right)}(x, y)+d^{\left(\delta_{1}\right)}\left(y, x_{0}\right)$. Hence for $\beta \geq \beta_{2}$ we have

$$
d_{\beta}(x, y) \geq d^{\left(\delta_{1}\right)}(x, y) .
$$

Now, let $\rho=d^{\prime}(x, y)$ [Recall that $d^{\prime}(\cdot, \cdot)$ is the distance function on $\Omega^{\prime}$ ]. Since the ball $\left\{z \in \Omega^{\prime} \mid d^{\prime}(x, z)<2 \rho\right\}$ is contained in $\Omega_{2 \rho}$, there exists a minimizing geodesic joining $x$ and $y$ and contained in $\Omega_{2 \rho}$. Hence further increasing $\Omega_{2 \rho}$ will not make the distance of $x$ and $y$ any smaller, that is

$$
d^{(2 \rho)}(x, y)=d^{(s)}(x, y)\left(=d^{\prime}(x, y)\right), \quad \text { all } s \geq 2 \rho .
$$

We have already assumed that $\beta$ is large enough so that $\beta^{-\eta} \leq \delta_{1} / 8$. Hence $\rho=d^{\prime}(x, y) \leq d(x, y) \leq \beta^{-\eta} \leq \delta_{1} / 2$ and therefore, by $(31), d^{(2 \rho)}(x, y)=d^{\left(\delta_{1}\right)}(x, y)$. Hence, using the fact that $2 \rho \leq 2 \beta^{-\eta}$ together with (H2), we have

$$
d_{\beta}(x, y) \geq d^{\left(\delta_{1}\right)}(x, y)=d^{(2 \rho)}(x, y) \geq d^{\left(2 \beta^{-\eta}\right)}(x, y) \geq\left(1-c \beta^{-v \eta}\right) d(x, y) .
$$

Combining this with (27) completes the proof upon choosing $\eta=1 /(1+v)$.

Remark. It follows from (30) that if $\beta$ is sufficiently large, if $x$ and $y$ are bounded away from $\partial \Omega$ and sufficiently close to each other, then $d_{\beta}(x, y)=d(x, y)$. Moreover, it is easily seen that hypotheses (H1) and (H2) are not needed in that case.

The next proposition provides a sufficient condition for $(\mathrm{H} 2)$ to be valid, with $\nu=1 / 2$. We conjecture that a more sophisticated way of retracting curves from $\Omega_{\delta}$ into $\bar{\Omega}$ would in fact yield the same result with $v=1$.

Given two curves $\gamma_{1}$ and $\gamma_{2}$ defined on $[a, b]$ and $[c, d]$ respectively and such that $\gamma_{1}(b)=\gamma_{2}(c)$, we denote by $\gamma_{1} \oplus \gamma_{2}$ the curve on $[a, b+d-c]$ which results from uniting $\gamma_{1}$ and $\gamma_{2}$, that is the curve that equals $\gamma_{1}(t)$ for $t \in[\alpha, \beta]$ and equals $\gamma_{2}(c-b+t)$ for $t \in[\beta, b+d-c]$.

Proposition 8. Let $\Omega$ be a bounded domain with $C^{\prime}$ boundary and let $p(\cdot, \cdot)$ be a Finsler metric in $\mathcal{F}_{1}(\Omega)$. Assume that $(\mathrm{H} 1)$ is satisfied. Then there exist constants $c, \delta_{0}>0$ such that for $\delta<\delta_{0}$ we have

for all $x, y \in \Omega$.

$$
\left(1-c \delta^{1 / 2}\right) d(x, y) \leq d^{(\delta)}(x, y) \leq d(x, y),
$$

Proof. We first prove the following

Claim. We have

$$
d^{\prime}(x, y)=p(x, y-x)+O\left(|y-x|^{2}\right), \quad(y \rightarrow x)
$$

uniformly in $x \in \mathbf{\Omega}$. 
Proof. Given $x \in \Omega$ and $\xi$ with $p(x, \xi)=1$, let $\gamma_{\xi}$ be the geodesic parametrized by arc length and satisfying $\gamma(0)=x, \dot{\gamma}(0)=\xi$. Then $\gamma(s)$ has a Lipschitz continuous second derivative and therefore

$$
\gamma_{\xi}(s)=\gamma_{\xi}(0)+s \dot{\gamma}(0)+O\left(s^{2}\right)
$$

where the $O$ is uniform in $\xi \in \mathbf{R}^{N}$ of unit length and in $x \in \Omega$. Let $y \in \Omega^{\prime}$ and near $x$ be given and let $\xi$ be such that $\gamma_{\xi}\left(d^{\prime}(x, y)\right)=y$. Substituting (and taking $s=d^{\prime}(x, y)$ ) we get

$$
y-x=d^{\prime}(x, y) \xi+O\left(|y-x|^{2}\right)
$$

and the claim follows by applying $p(x, \cdot)$ to both sides.

The assumption $\partial \Omega \in C^{l}$ means that there exist closed rectangles $V_{1}, \ldots, V_{s}$ that cover $\partial \Omega$ and have the following properties: each rectangle $V_{l}$ has the form $V_{l}=U_{l} \times[-\eta, \eta]$ for some $(N-1)$-dimensional rectangles $U_{l}$ and some small, but fixed, number $\eta$ independent of $l \in\{1, \ldots, r\}$; using local coordinates $\left(z^{\prime}, z_{N}\right)$ on each $V_{l}$ (so $z^{\prime} \in U_{l}$ and $\left.z_{N} \in[-\eta, \eta]\right)$ there exist $C^{l}$ functions $\rho_{l}$ defined on $U_{l}$ and such that

$$
\begin{aligned}
\partial \Omega \cap V_{l} & =\left\{\left(z^{\prime}, z_{N}\right) \mid z_{N}=\rho_{l}\left(z^{\prime}\right)\right\}, & & l=1, \ldots, r, \\
\Omega \cap V_{l} & =\left\{\left(z^{\prime}, z_{N}\right) \mid z_{N}>\rho_{l}\left(z^{\prime}\right)\right\}, & & l=1, \ldots, r .
\end{aligned}
$$

(There is a certain inconsistency in this notation since $z_{N}$ also denotes the $N$ th Euclidean coordinate.) We shall call the axis of $z_{N}$ the normal axis for the rectangle. By making the rectangles smaller if necessary, we may assume that the angle between the normal axis of each rectangle $V_{1}$ and the (inward) normal at each $z \in V_{1} \cap \partial \Omega$ does not exceed a small fixed number, such as $\pi / 100$.

There exists $\delta_{0}>0$ such that if $d^{\prime}(x, y)<\delta_{0}$ and if the distances of both $x$ and $y$ from $\partial \Omega$ is smaller than $\delta_{0}$, then there exists a rectangle $V=U \times[-\eta, \eta]$ in the above collection that contains both $x$ and $y$; we assume that $x$ and $y$ are such and write $x=\left(x^{\prime}, x_{N}\right), y=\left(y^{\prime}, y_{N}\right)$ in the local coordinates of $V$. Let $\rho$ be the function on $U$ which represents $\partial \Omega$ in $V$. Then the numbers $\alpha:=x_{N}-\rho\left(x^{\prime}\right)$ and $\beta:=y_{N}-\rho\left(y^{\prime}\right)$ are both positive. We define a curve $\gamma$ by

$$
\gamma(t)=\left(x^{\prime}+t\left(y^{\prime}-x^{\prime}\right), \rho\left(x^{\prime}+t\left(y^{\prime}-x^{\prime}\right)\right)+\alpha+t(\beta-\alpha)\right), \quad t \in[0,1]
$$

and note that it lies entirely in $\Omega$ and satisfies $\gamma(0)=x$ and $\gamma(1)=y$. We also have

$$
\dot{\gamma}(t)=\left(y^{\prime}-x^{\prime},\left(y^{\prime}-x^{\prime}\right) \cdot(\nabla \rho)\left(x^{\prime}+t\left(y^{\prime}-x^{\prime}\right)\right)+(\beta-\alpha)\right) .
$$

But $\left(y^{\prime}-x^{\prime}\right) \cdot(\nabla \rho)\left(x^{\prime}+t\left(y^{\prime}-x^{\prime}\right)\right)=\rho\left(y^{\prime}\right)-\rho\left(x^{\prime}\right)+O\left(\left|y^{\prime}-x^{\prime}\right|^{2}\right)$, where the big 0 is uniform with respect to $x$ and $y$ (and $t$ ) by a simple compactness argument. Hence 


$$
\dot{\gamma}(t)=y-x+O\left(|y-x|^{2}\right) .
$$

From this follows easily that

$$
p(\gamma(t), \dot{\gamma}(t))=p(x, y-x)+O\left(|y-x|^{2}\right),
$$

and therefore

$$
d(x, y) \leq \int_{0}^{1} p(\gamma(t), \dot{\gamma}(t)) d t \leq p(x, y-x)+c|y-x|^{2} .
$$

Recalling (33) we conclude that $d(x, y) \leq d^{(\delta)}(x, y)+c|y-x|^{2}$ and therefore, using the equivalence with the Euclidean distance, that

$$
d(x, y) \leq d^{(\delta)}(x, y)\left(1+c \delta^{1 / 2}\right)
$$

for all $x$ and $y$ in $\Omega$ that satisfy $d(x, y) \leq \delta^{1 / 2}$, provided $\delta$ is sufficiently small, smaller than $\delta_{0}$, say. Hence (32) is proved in the case $d(x, y) \leq \delta^{1 / 2}$. We next consider the complementary case.

Let $\gamma(t), t \in[0,1]$, be a path contained in $\Omega_{\delta}$ and joining the points $x, y \in \Omega$. We define the closed intervals $I_{i}=\left[\alpha_{i}, \beta_{i}\right], i=1, \ldots, r$, by requiring that (i) they are disjoint (ii) $\alpha_{i+1}>\beta_{i}$ and (iii) $\gamma(t) \in \Omega_{\delta} \backslash \Omega$ iff $t \in \cup_{i=1}^{r} I_{i}$. Let $\gamma_{i}=\left.\gamma\right|_{I_{i}}, i=1, \ldots, r$. The trace of each $\gamma_{i}$ then lies entirely in $\cup_{l=1}^{s} V_{l}$ and outside $\Omega$. For every $i=1, \ldots, r$ we choose a finite sequence $\left(V_{i, 1}, V_{i, 2}, \ldots, V_{i, r_{1}}\right)$, contained, as a set, in $\left\{V_{l}\right\}_{l=1}^{s}$, and such that (i) $\gamma\left(\alpha_{i}\right) \in V_{i, 1}$ and (ii) each $V_{i, j}$ is added to the sequence when $\gamma_{i}$ enters its interior. This implies in particular that $\gamma\left(\beta_{i}\right) \in V_{i, r}$. (If $\gamma$ enters two or more rectangles at the same time, we simply choose one of them.) This induces a partition

$$
\alpha_{i}=: \beta_{i, 0}<\beta_{i, 1}<\ldots<\beta_{i, r_{i-1}}<\beta_{i, r_{i}}:=\beta_{i}
$$

of the interval $I_{i}$ by setting

$$
\beta_{i, j}=\inf \left\{t \in I_{i} \mid \gamma(t) \in V_{i, j+1}\right\}, \quad j=1, \ldots, r_{i}-1 .
$$

Let $I_{i, j}=\left[\beta_{i, j-1}, \beta_{i, j}\right], j=1, \ldots, r_{i}$, and let $\gamma_{i, j}=\left.\gamma\right|_{I_{i, j}}$. Then (the trace of) each $\gamma_{i, j}$ lies entirely in the rectangle $V_{i, j}$. For $\delta$ sufficiently small we define a new curve $\theta_{i, j}$ by shifting the curve $\gamma_{i, j}$ by $2 \delta / k_{1}$ (where $k_{1}$ is as in (13)) in the direction of the normal axis of the rectangle $V_{i, j}$. That is, if the curve $\gamma_{i, j}$ is given, in the local coordinates of $V_{i, j}$, by $\gamma_{i, j}(t)=(u(t), v(t))$, we define

$$
\theta_{i, j}(t)=\left(u(t), v(t)+2 \delta / k_{1}\right) .
$$

If $z \in \Omega_{\delta} \backslash \Omega$, then the Euclidean distance of $z$ from $\Omega$ is not greater than $\delta / k_{1}$ by (13). Hence, from our assumption that the normal axis of $V_{i, j}$ is very close to the inward 
normal at any point of $V_{i, j} \cap \partial \Omega$, it follows that $\theta_{i, j}$ is a curve contained in $\Omega$ and we also have, since $\left|\theta_{i, j}-\gamma_{i, j}\right|=2 \delta / k_{1}$,

$$
\left|l\left(\theta_{i, j}\right)-l\left(\gamma_{i, j}\right)\right| \leq \int_{I_{i, j}}\left|p\left(\theta_{i, j}, \dot{\gamma}_{i, j}\right)-p\left(\gamma_{i, j}, \dot{\gamma}_{i, j}\right)\right| d t \leq c \delta l\left(\gamma_{i, j}\right)
$$

Now, let $s_{i, j}, j=1, \ldots, r_{i}-1$, be the straight segments that join $\theta_{i, j}\left(\beta_{i, j}\right)$ and $\theta_{i, j+1}\left(\beta_{i, j}\right)$. The Euclidean length of each $s_{i, j}$ does not exceed $4 \delta / k_{1}$ by the triangle inequality. Then

$$
\theta_{i}:=\theta_{i, 1} \oplus s_{i, 1} \oplus \theta_{i, 2} \oplus s_{i, 2} \oplus \cdots \oplus s_{i, r_{i}-1} \oplus \theta_{i, r_{i}}
$$

is an absolutely continuous curve since the initial point of each curve in the string coincides with the final point of the previous one. Moreover, for $i=1, \ldots, r$,

$$
\begin{aligned}
\left|l\left(\theta_{i}\right)-l\left(\gamma_{i}\right)\right| & \leq \sum_{j=1}^{r_{i}}\left|l\left(\theta_{i, j}\right)-l\left(\gamma_{i, j}\right)\right|+\sum_{j=1}^{r_{i}-1} l\left(s_{i, j}\right) \\
& \leq c \delta \sum_{j=1}^{r_{1}} l\left(\gamma_{i, j}\right)+c r_{i} \delta \\
& =c \delta l\left(\gamma_{i}\right)+c r_{i} \delta .
\end{aligned}
$$

Now, let $J_{0}=\left[0, \alpha_{1}\right], J_{i}=\left[\beta_{i}, \alpha_{i+1}\right], i=1, \ldots, r-1, J_{r}=\left[\alpha_{r}, 1\right]$ and let $\zeta_{i}=\left.\gamma\right|_{J_{i}}$. For each $i=1, \ldots, r$ we consider straight segments $s_{i}$ and $s_{i}^{\prime}$ by means of which we connect (i) the final point of the curve $\zeta_{i-1}$ to the initial point of the curve $\theta_{i}$ and (ii) the final point of the curve $\theta_{i}$ to the initial point of the curve $\zeta_{i}$. Each one of these segments has Euclidean length equal to $2 \delta / k_{1}$ by construction. We then define the curve

$$
\theta:=\zeta_{0} \oplus s_{1} \oplus \theta_{1} \oplus s_{1}^{\prime} \oplus \zeta_{1} \oplus \cdots \oplus s_{r} \oplus \theta_{r} \oplus s_{r}^{\prime} \oplus \zeta_{r} .
$$

The curve $\theta$ is then absolutely continuous and, by construction, lies entirely in $\Omega$ and has the same endpoints as $\gamma$. Moreover, since $\gamma=\zeta_{0} \oplus \gamma_{1} \oplus \zeta_{1} \oplus \gamma_{2} \oplus \cdots \oplus \zeta_{r-1} \oplus \gamma_{r} \oplus \zeta_{r}$, we have

$$
\begin{aligned}
|l(\theta)-l(\gamma)| & \leq \sum_{i=1}^{r}\left|l\left(\theta_{i}\right)-l\left(\gamma_{i}\right)\right|+\sum_{i=1}^{r}\left\{l\left(s_{i}\right)+l\left(s_{i}^{\prime}\right)\right\} \\
& \leq c \delta \sum_{i=1}^{r} l\left(\gamma_{i}\right)+c \delta \sum_{i=1}^{r} r_{i}+c r \delta \\
& \leq c \delta l(\gamma)+c r \delta\left[1+\max _{1 \leq i \leq r} r_{i}\right] .
\end{aligned}
$$

Hence 
608

\section{G. BARBATIS}

$$
l(\theta) \leq(1+c \delta) l(\gamma)+c r \delta\left[1+\max _{1 \leq i \leq r} r_{i}\right]
$$

Now, let $\left(\gamma_{n}\right)$ be a sequence of absolutely continuous curves in $\Omega_{\delta}$ such that

$$
l\left(\gamma_{n}\right)<d^{(\delta)}(x, y)+(1 / n)
$$

To each $\gamma_{n}$ correspond numbers $r(n)$ and $r_{i}(n)$ as described above. A simple application of the Arzela-Ascoli theorem shows that, by replacing $\left(\gamma_{n}\right)$ by a subsequence if necessary, there exists a constant $c_{1}$ such that

$$
\sup _{n} r(n)<c_{1}, \quad \sup _{n} r_{i}(n)<c_{1}
$$

Moreover, the constant $c_{1}$ can be taken to be independent of the endpoints $x$ and $y$ by the compactness of $\bar{\Omega}$. Now, let $\theta_{n}$ be curves in $\Omega$ produced from the $\gamma_{n}$ 's in the way described above. It then follows from (35) and (37) that there exists a constant $c$ such that

$$
l\left(\theta_{n}\right) \leq(1+c \delta) l\left(\gamma_{n}\right)+c \delta, \quad n=1,2, \ldots
$$

Combining this with (36) yields

$$
d(x, y) \leq l\left(\theta_{n}\right) \leq(1+c \delta)\left[d^{(\delta)}(x, y)+(1 / n)\right]+c \delta
$$

and hence, letting $n \rightarrow \infty$,

$$
d(x, y) \leq(1+c \delta) d^{(\delta)}(x, y)+c \delta
$$

for all $\delta$ sufficiently small and all $x, y \in \Omega$. This implies (32) when $d(x, y) \geq \delta^{1 / 2}$.

\section{REFERENCES}

1. M. Abate and G. Patrizo, Finsler metrics - a global approach (Lecture Notes in Mathematics, 1591, Springer, 1994).

2. S. AGmon, Lectures on exponential decay of solutions of second-order elliptic equations (Mathematical Notes, Princeton University Press, 1982).

3. P. Auscher, T. Coulhon and P. Tchamitchian, Absence de principe du maximum pour certaines équations paraboliques complexes, Colloq. Math., to appear.

4. G. Barbatis and E. B. Davies, Sharp bounds on heat kernels of higher-order uniformly elliptic operators, J. Operator Theory 36 (1996), 179-198.

5. G. BARBAtis, Sharp heat kernel bounds and Finsler-type metrics, Quart. J. Math. Oxford Ser. (2) 49 (1998), 261-277.

6. A. BejanCU, Finsler geometry and applications (Ellis Horwood, 1990).

7. W. M. Bоoтнву, An introduction to differentiable manifolds and Riemannian geometry (Academic Press, 1986). 
8. H. BuSEMANN, Metric methods in Finsler spaces and in the foundations of geometry (Princeton University Press, 1942).

9. E. B. Davies, Explicit constants for Gaussian upper bounds on heat kernels, Amer. J. Math. 109 (1987), 319-334.

10. E. B. Davies, Uniformly elliptic operators with measurable coefficients, J. Funct. Anal. 132 (1995), 141-169.

11. E. B. Davies, $L^{p}$ spectral theory of higher-order elliptic differential operators, Bull. London Math. Soc. 29 (1997), 513-546.

12. D. E. EDMunds and W. D. Evans, Spectral theory and differential operators (Oxford University Press, 1987).

13. M. A. Evgrafov and M. M. Postnikov, Asymptotic behavior of Green's functions for parabolic and elliptic equations with constant coefficients, Math. USSR-Sb. 11 (1970), 1-24.

14. M. A. Evgrafov and M. M. Postnikov, More on the asymptotic behavior of the Green's functions of parabolic equations with constant coefficients, (Russian) Mat. Sb. 92 (134) (1973), 171-194.

15. M. OWEN, A Riemannian off-diagonal heat kernel bound for uniformly elliptic operators, preprint 1997.

16. D. W. Robinson, Elliptic operators and Lie groups (Oxford University Press, London, New York, 1991).

17. H. RuND, The differential geometry of Finsler spaces (Springer-Verlag, 1959).

18. K. Tintarev, Short time asymptotics for fundamental solutions of higher order parabolic equations, Comm. Partial Differential Equations 7 (1982), 371-391.

Department of Mathematics

King's COLLEGE

STRAND

LONDON WC2R 2LS

ENGLAND

Current address:

Department of Mathematics

UNIVERSITY OF CRETE

71409 HERAKLION

CreTe

GREeCE 